\title{
Dados Abertos Governamentais: usuários e apropriações socials no BrasiL ${ }^{1}$
}

\author{
Maria Dominguez Costa Pinho²
}

\section{INTRODUÇÃO}

Há pelo menos uma década, desde a implementação das primeiras políticas públicas de governo aberto e de dados abertos por governos ao redor do mundo, e com a adoçáo de tecnologias, ferramentas e dispositivos de comunicação digital por parte das administraçôes públicas, temos visto crescer exponencialmente o volume de dados e informaçôes públicas à disposição dos cidadãos.

Nesse contexto, o governo federal brasileiro adotou, nos últimos anos, uma série de políticas e iniciativas de dados abertos governamentais, evidenciadas, por exemplo, pelo envolvimento do país com a Parceria para Governo Aberto (Open Government Partnership), desde a sua concepção, em 2011, como país cofundador. A acessibilidade dos dados abertos passou a ser amparada pela Lei de Acesso à Informação (LAI), via transparência passiva. A regulamentação do Decreto no 8.777/2016 foi responsável, ainda, por editar a Política de Dados Abertos do Poder Executivo federal, criar a Infraestrutura Nacional de Dados Abertos e tornar obrigatória a criação de Planos de Dados Abertos (PDAs) por cada órgão público federal. Por fim, nota-se que houve um aumento da quantidade de bases de dados publicadas no Portal Brasileiro de Dados Abertos. ${ }^{3}$

Inúmeras expectativas associam-se aos usos e às apropriaçóes sociais de dados abertos por atores como jornalistas, gestores públicos, cientistas, programadores, ativistas, organizaçóes não governamentais (ONGs), entre outros. Não só por meio da sua publicação, mas principalmente pelo seu uso efetivo, espera-se, por exemplo, que seja produzida mais transparência, participação e colaboração nos negócios públicos, além do desenvolvimento social e econômico. Falta ainda, todavia, uma noção de quem sáo os usuários de dados abertos e quais usos sáo feitos desses dados no Brasil.

\section{SOBRE DADOS ABERTOS GOVERNAMENTAIS}

Dados abertos governamentais podem ser definidos como dados públicos, originados das funçóes administrativa e governamental, disponíveis em um formato aberto, isto é, legíveis por máquinas, não proprietários, não discriminatórios e com licenças abertas específicas que garantam a sua reutilização. ${ }^{4}$ Aqui, considera-se, por exemplo, que informaçôes governamentais orçamentárias, geográficas, sociais, demográficas, legislativas, ambientais, meteorológicas, entre outras, que são produzidas diariamente no cumprimento das atividades públicas, no âmbito dos diferentes setores e órgãos de governo, podem ser formatadas e tornadas acessíveis como dados abertos governamentais, por serem dotadas de valor e interesse público.

1. DOI: http://dx.doi.org/10.38116/bapi25art3

2. Pesquisadora associada no Instituto Nacional de Ciência e Tecnologia em Democracia Digital (INCT.DD).E-mail:<mariadominguezcp@gmail.com>. 3. Em 17 de janeiro de 2021, o portal contava com um volume de 10.688 conjuntos de dados disponíveis ao acesso público. A dissertação de mestrado da autora deste artigo demonstra que, em dezembro de 2018, esse número equivalia a 6.317 conjuntos. 0 portal está disponível em: <https://dados.gov.br/>.

4. Entre os principais requisitos técnicos dos dados abertos, estão os Oito Princípios dos Dados Abertos Governamentais. Mais informações sobre cada um desses princípios estão disponíveis em: $<$ https://opengovdata.org/>. 
O acesso irrestrito a um grande volume de conjuntos de dados 5 públicos que antes não eram acessíveis parece, por si só, ser um facilitador de inúmeros benefícios, associados, inclusive, a princípios democráticos clássicos, como transparência e participação. Sendo assim, a maior parte da literatura sobre dados abertos governamentais os define, principalmente, como promotores de mais transparência e accountability (Geiger e Lucke, 2012; Janssen, 2011; Lourenço, 2015) e de envolvimento e participação dos cidadãos nos negócios públicos (Conradie e Choenni, 2014; Evans e Campos, 2013), além de promoverem a colaboraçáo e cocriaçáo entre setores sociais e governos (Noveck, 2015).

Dados abertos governamentais são conhecidos por serem os maiores propulsores de governos abertos (Kučera, Chlapek e Nečaský, 2013). Além das expectativas associadas aos princípios democráticos, há, ainda, aquelas concernentes à eficiência e eficácia dos governos: dados abertos podem levar tomadores de decisão política, gestores públicos, analistas e grupos de interesse a produzir políticas e serviços públicos melhores e mais precisos (Janssen, Charalabidis e Zuiderwijk, 2012; Clarke e Margetts, 2014).

O uso de dados abertos, enfim, é associado à oferta de novas possibilidades para o desenvolvimento econômico e social, pela capacidade de promover estímulo econômico por meio da criaçáo de novos produtos e serviços (Zeleti, Ojo e Curry, 2016; Lindman, 2014), além da possibilidade de gerar novos modelos de negócios mediante a colaboração entre atores públicos e privados (Howard, 2013).

Em contrapartida, o uso massivo de dados digitais e abertos por parte dos governos apresenta possibilidades de vigilância e violação da privacidade dos cidadãos jamais vistas antes (Kitchin, 2014). Nesse cenário, reforça-se a necessidade de políticas de proteção de dados pessoais dialogarem com políticas de transparência e dados abertos, reconhecendo ambas como complementares, não excludentes, e como direitos fundamentais dos cidadáos.

Apesar das dificuldades de mensurar os benefícios supracitados associados à oferta e ao uso de dados abertos governamentais, argumentamos que, para tomar conhecimento dos resultados que podem ser extraídos de políticas públicas como essa é preciso, primeiramente, compreender quem são os usuários e que tipos de usos são feitos desses dados. Zuiderwijk (2015) reforça essa visão constatando que, normalmente, o uso que se faz dos dados abertos é uma questáo menosprezada pelos próprios governos, mais interessados na publicaçáo de tais dados.

\section{PROCEDIMENTOS METODOLÓGICOS}

Tendo como objetivo lançar luz sobre os usuários de dados abertos no Brasil, o estudo em tela consiste em uma pesquisa exploratória, seguindo alguns procedimentos metodológicos. Primeiro, um questionário foi aplicado em grupos do WhatsApp, Facebook e Telegram chamados Dados Abertos Brasil, formados por participantes interessados no tema, oriundos de diferentes ocupaçóes profissionais. O questionário também foi compartilhado nos perfis do Facebook e Twitter do instituto de pesquisa do qual a autora deste estudo faz parte e ficou disponível aos participantes entre novembro e dezembro de 2018. A pesquisa foi aplicada também no III Encontro Brasileiro de Governo Aberto, em São Paulo, realizado nos dias 4 e 5 de dezembro do mesmo ano. Para garantir

5. Por conjunto de dados ou base de dados, entende-se um agregado de dados relacionados entre si, por grupo temático, por exemplo. Cada conjunto de dados pode ter um ou mais recursos. Um exemplo em destaque no Portal Brasileiro de Dados Abertos é a base referente às Compras Públicas do Governo Federal, disponível em: <http://www.dados.gov.br/dataset/compras-publicas-do-governo-federal>. 
que todos os participantes da pesquisa já tivessem sido, pelo menos uma vez, usuários primários ${ }^{6}$ de dados abertos, foram eliminados da amostra todos aqueles que responderam negativamente à seguinte pergunta: você já utilizou dados abertos governamentais alguma vez? Assim, esta pesquisa chegou a uma amostra composta por 81 respostas. Esta amostra não é probabilística e, portanto, não representa o universo de usuários de dados abertos do país.

Em seguida, com o intuito de levantar dados qualitativos, que auxiliam a interpretação dos dados coletados mediante questionário, cinco entrevistas em profundidade ${ }^{7}$ foram conduzidas com usuários de dados abertos. Cada um dos entrevistados corresponde a um perfil profissional, sendo eles: i) gestor público (Controladoria-Geral da União - CGU); ii) membro de uma ONG (Open Knowledge Foundation Brasil); iii) jornalista de dados (Estadáo Dados); iv) desenvolvedor (Brasil.IO); e v) pesquisador (Diretoria de Análise de Políticas Públicas da Fundação Getulio Vargas (DAPP/FGV). A seleção dos entrevistados foi realizada de acordo com o conhecimento prévio sobre a relevância dos trabalhos de cada um com dados abertos ou pela indicação de outro entrevistado (técnica conhecida como "bola de neve").

\section{USUÁRIOS DE DADOS ABERTOS GOVERNAMENTAIS, USOS E APROPRIAÇÕES SOCIAIS NO BRASIL}

De acordo com outros estudos sobre usuários de dados abertos governamentais, no Brasil, identificou-se que essas partes interessadas também são formadas por jornalistas, pesquisadores, desenvolvedores, gestores públicos e membros de ONGs (Bates, 2012; Gonzalez-Zapata e Heeks, 2015; Baack, 2015; Safarov, Meijer e Grimmelikhuijsen, 2017). O maior grupo de usuários de dados abertos identificado por esta pesquisa foi aquele cujos integrantes declararam ser pesquisadores(as) $(\mathrm{N}=18)$, seguido do grupo de desenvolvedores(as) $(\mathrm{N}=16)$, gestores(as) públicos(as) $(\mathrm{N}=12)$, cientistas de dados $(\mathrm{N}=12)$, jornalistas $(\mathrm{N}=11)$, membros de $\mathrm{ONG}(\mathrm{N}=6)$, ativistas $(\mathrm{N}=3)$ ou de outras profissóes $(\mathrm{N}=3)$, identificadas como social media, tradutor e empresário.

Quanto ao perfil sociodemográfico dos entrevistados, os usuários de dados abertos contemplados por este estudo têm, como faixa etária proeminente, as idades entre 30 e 34 anos $(\mathrm{N}=21$ ou $26 \%$ da amostra). Além disso, $68 \%$ da amostra $(\mathrm{N}=55)$ demonstrou se identificar com o gênero masculino e $30 \%(\mathrm{~N}=25)$, com o feminino. Uma pessoa declarou não se identificar com nenhum dos dois gêneros. Quanto ao nível de escolaridade máximo atingido, 52\% indicaram ter pós-graduação completa $(\mathrm{N}=42)$. A amostra é composta por $73 \%$ de participantes que identificaram sua cor da pele como branca $(\mathrm{N}=59)$. Enfim, em relação à renda mensal familiar, 41\% da amostra declarou receber entre R \$ 3.150 e R \$ 7.880 $(\mathrm{N}=33)$. Estamos, portanto, diante de uma amostra superescolarizada, náo representativa em termos de cor da pele e gênero e com renda familiar não correspondente à distribuição da renda familiar no Brasil.

Foi perguntado qual a importância do uso de dados abertos para as atividades rotineiras desempenhadas pelos entrevistados em suas ocupaçóes profissionais. $\mathrm{O}$ gráfico 1 demonstra que a maior parte dos participantes da pesquisa conferiu grande importância ao uso de dados abertos para os seus setores profissionais, classificando-o, principalmente, como importante e muito importante. Considerando as limitaçóes dessa amostra, os pesquisadores, gestores públicos e desenvolvedores são aqueles que atribuem maior importância aos dados abertos para a execução das suas tarefas,

6. Neste trabalho, fazemos uma distinção entre usuários de dados primários e secundários. Usuários primários são aqueles que têm acesso à base de dados do modo como ela foi publicada pelos órgãos públicos. São estes usuários que realizam download, organizam e manipulam os dados. Compreendemos como usuários secundários aqueles que têm acesso apenas aos produtos e iniciativas criados com os dados, como reportagens, pesquisas, aplicativos ou interfaces de visualização de dados.

7. A transcrição das entrevistas em profundidade e o questionário podem ser encontrados, na íntegra, em Pinho (2019). 
classificando-os em maior porcentagem como muito importantes para o cumprimento de suas funçóes, como exibe a tabela 1.

\section{GRÁFICO 1}

Grau de importância do uso de dados abertos, por profissão

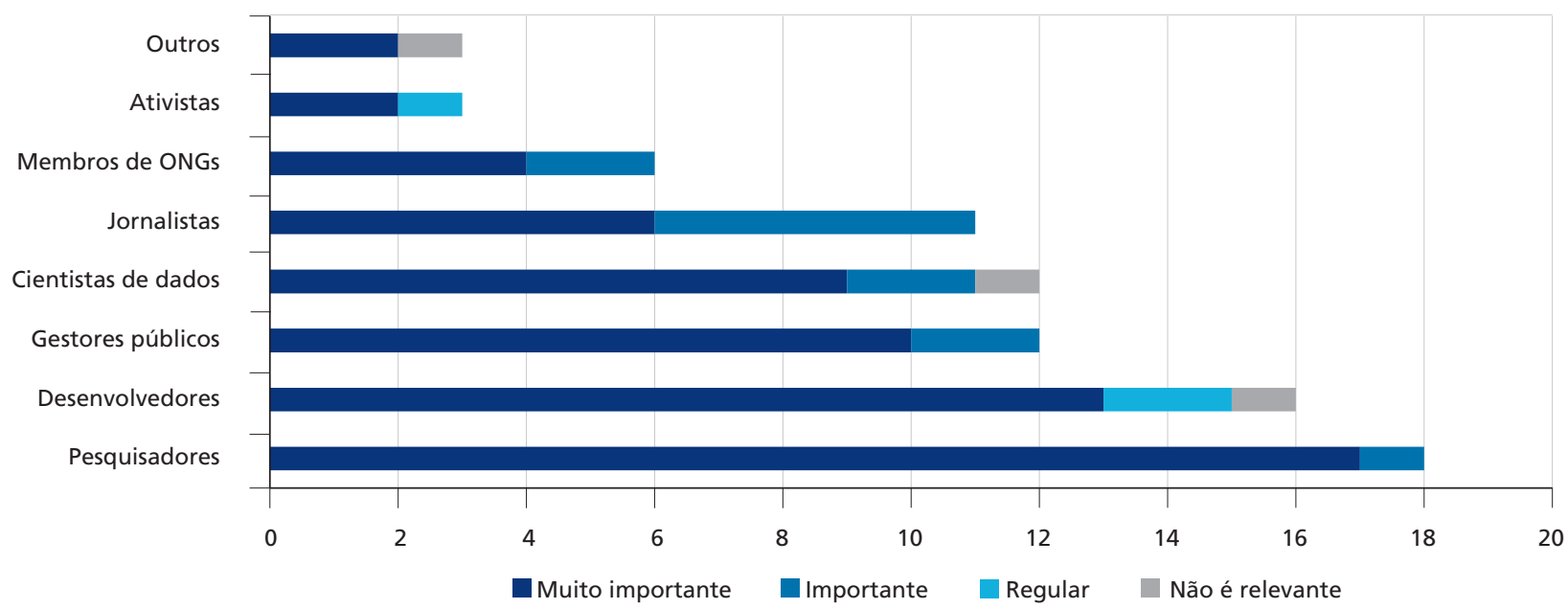

Elaboração da autora.

TABELA 1

Distribuição dos entrevistados que consideram o uso de dados abertos muito importante para o seu trabalho

\begin{tabular}{ll}
\hline Entrevistados & $\%$ \\
\hline Pesquisadores & 94 \\
Gestores públicos & 83 \\
Desenvolvedores & 81 \\
Cientistas de dados & 75 \\
ONGs, ativistas e outros & 67 \\
Jornalistas & 54 \\
\hline
\end{tabular}

Elaboração da autora.

Apesar de não figurarem, aqui, como entrevistados que apontaram o uso de dados como mais relevante para as suas profissóes, há, no Brasil, programas de treinamento desenvolvidos especificamente para a capacitação de jornalistas e organizaçóes da sociedade civil para o uso de dados abertos. Vimos crescer, também, a quantidade de jornalistas que trabalham com dados no país. ${ }^{8}$ Para o profissional membro da ONG entrevistado por esta pesquisa, estima-se que um dos maiores benefícios do uso de dados abertos no Brasil seja justamente a produção de um jornalismo de melhor qualidade.

Temos o fortalecimento do jornalismo de dados, que tem a informação como matéria-prima do seu trabalho, e se beneficia dessa abertura de dados. E temos a possibilidade de jornalistas, programadores e organizaçôes da sociedade civil realizarem o monitoramento e a análise de políticas públicas, para que todo o esforço de implementar ações e programas governamentais não se perca (entrevistado 2).

8. De-Lima-Santos (2019), em seu artigo Tem \#DDJBR aqui? Mapeando a presença do jornalismo de dados no Brasil, identifica que atualmente existem 52 organizações que trabalham com jornalismo de dados no país. 
Se, por um lado, os dados abertos passaram a ser recursos relevantes para as profissóes supracitadas, por outro, os entrevistados identificaram inúmeras barreiras associadas ao seu uso. Destacamos que, primeiramente, há uma barreira para o uso desses dados no que se refere aos cidadãos comuns, já que para utilizá-los são requeridos conhecimentos básicos de análises, mineração e visualização de dados (Zuiderwijk, 2015), além de, muitas vezes, noçóes de estatística, programação e design. Em consonância com os resultados obtidos por este estudo, vimos que o ecossistema de usuários de dados abertos envolve alguns usuários específicos, e o cidadão comum que não detém essas competências quase não tem participação direta nesse tipo de iniciativa (Safarov, Meijer e Grimmelikhuijsen, 2017; Sandoval-Almazan e Styrin, 2018).

Em segundo lugar, ainda no que se refere às barreiras identificadas aos usos de dados abertos, ${ }^{9}$ para os participantes desta pesquisa a principal dificuldade diz respeito à baixa qualidade dos dados publicados por órgãos governamentais, como ilustra o gráfico 2. Por conjuntos de dados com baixa qualidade entende-se aqueles que estão incompletos, desatualizados, sem metadados, desestruturados ou que não cumprem os requisitos técnicos exigidos para serem considerados abertos. Em alguns casos, essas falhas podem acarretar até mesmo a impossibilidade de reúso dos dados. O desenvolvedor que participou da entrevista em profundidade deste estudo, por exemplo, trabalha com uma iniciativa ${ }^{10}$ que propóe melhorias à qualidade das bases de dados governamentais, nem sempre publicadas em formatos adequados.

A ideia do projeto é pegar os dados que não estão acessíveis e disponibilizá-los em um formato palatável para pessoas que não têm conhecimento de programação. (...) Tornar os dados abertos inacessíveis é de certa forma elitizar a democracia, pois significa restringir o acesso aos dados. O que o Brasil.IO tenta fazer é tirar esse privilégio dos programadores para que todo mundo consiga usar esses dados (entrevistado 4).

\section{GRÁFICO 2}

\section{Barreiras encontradas no uso de dados abertos governamentais}

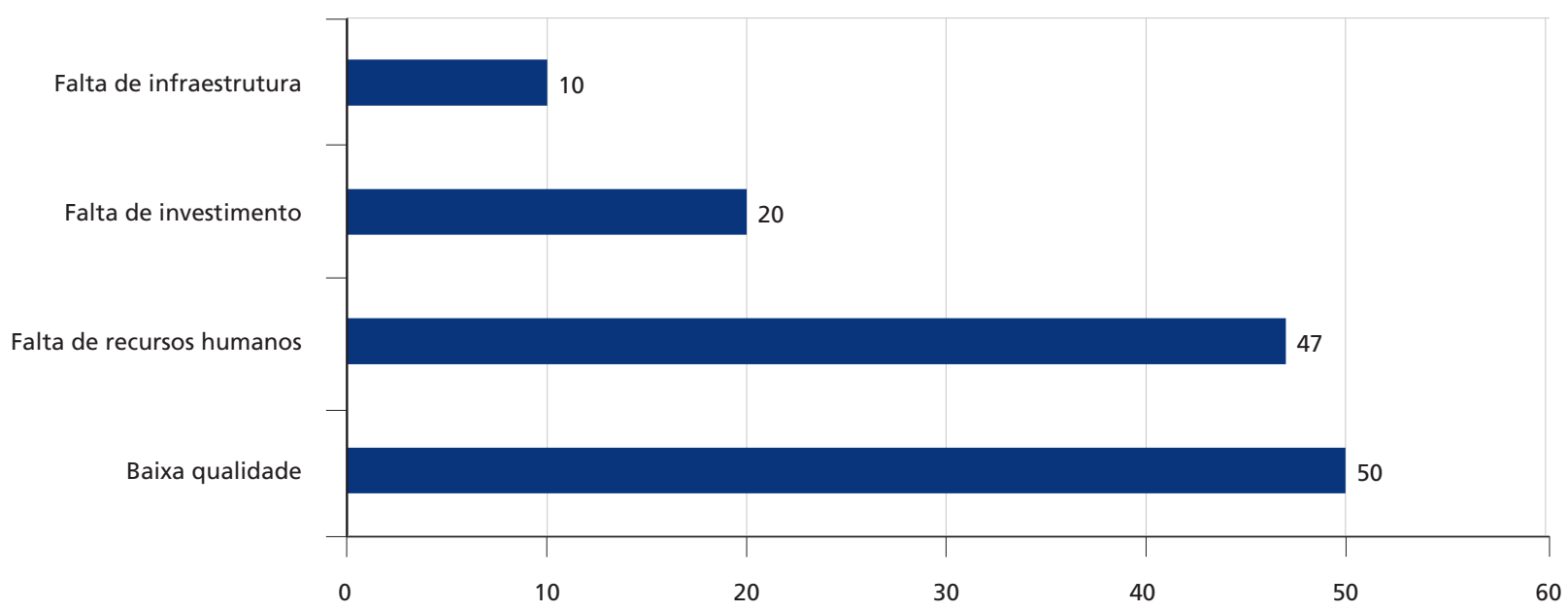

Elaboração da autora.

Outras barreiras identificadas foram: a falta de recursos humanos capacitados para trabalhar com dados abertos dentro das organizaçóes que abrigam os usuários de dados identificados por este questionário; a falta de investimento dessas organizaçóes, como em capacitaçôes e em recursos técnicos; e a falta de infraestrutura necessária para trabalhar com dados, como programas e softwares específicos.

9. Os entrevistados puderam assinalar mais de uma barreira associada ao uso de dados abertos.

10. A iniciativa, chamada Brasil.IO, está disponível em: <https://brasil.io/home>. 
Buscamos identificar, ainda, se há maior interesse em bases de dados oriundas de determinada área de atuação governamental. A maioria dos entrevistados afirmou buscar, principalmente, dados sobre educação, orçamento público e saúde. Todavia, vemos que há uma demanda por abertura de dados em diversas outras áreas e órgáos públicos.

\section{GRÁFICO 3}

Áreas de interesse dos entrevistados, por setores governamentais

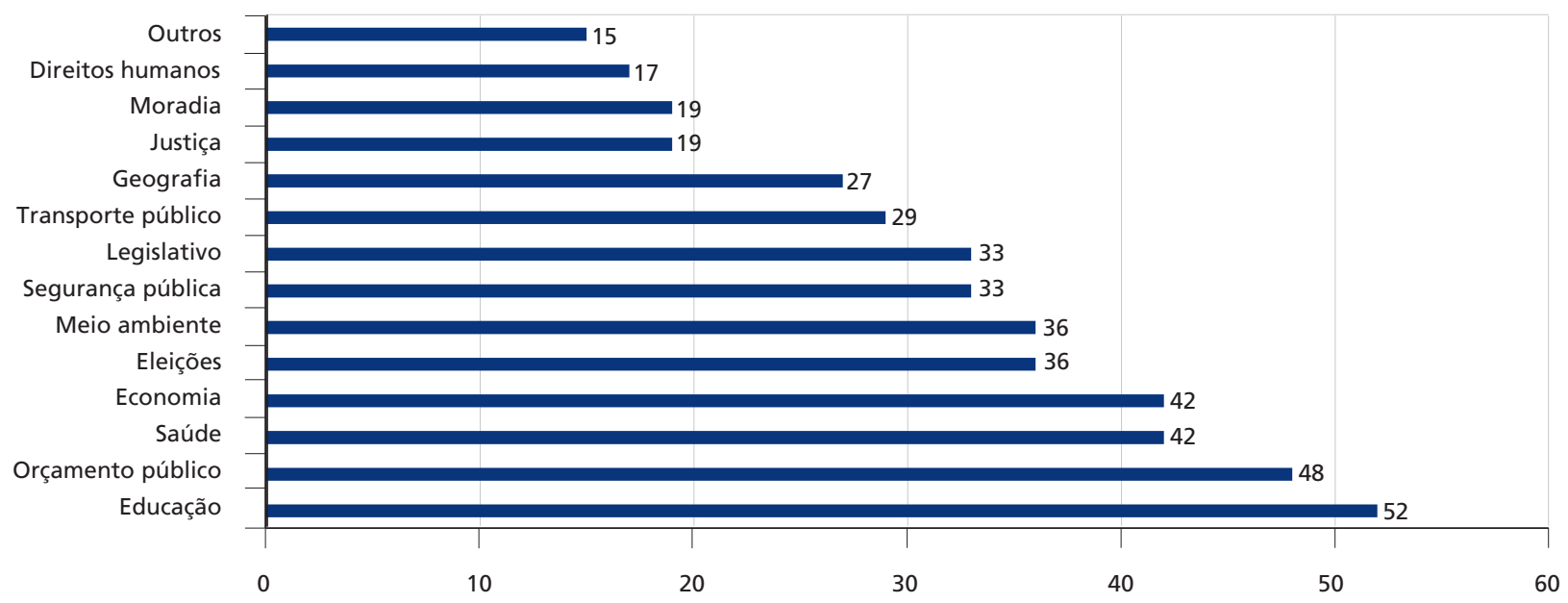

Elaboração da autora.

Enfim, reconhecendo a publicação de um grande volume de bases de dados por parte do Executivo federal brasileiro, principalmente desde a obrigação legal dos órgãos federais de planejarem a abertura de dados por meio dos PDAs, foi perguntado aos entrevistados quais sáo os meios de acesso aos dados de seus interesses. O gráfico 4 mostra que a maioria usa como fonte portais de órgãos específicos, mas os dados são acessados também no Portal da Transparência, via transparência passiva (pedidos de acesso via LAI), e, apenas em último lugar, por meio do Portal Brasileiro de Dados Abertos.

\section{GRÁFICO 4}

\section{Fontes de acesso aos dados abertos}

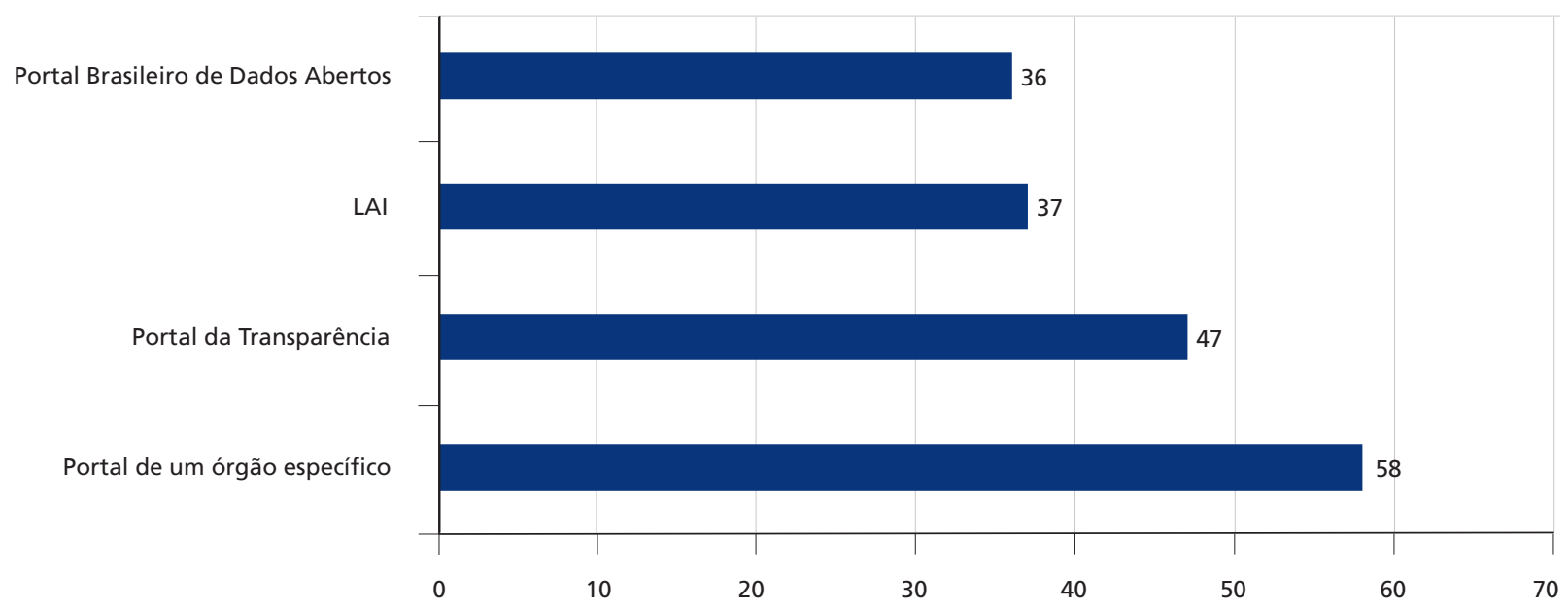

Elaboração da autora. 
O Portal Brasileiro de Dados Abertos foi criado em 2012 com o objetivo de se tornar mais amplo que o Portal da Transparência, ao promover o acesso a dados abertos de qualquer órgão público que deseje publicar seus dados, extrapolando, por exemplo, o governo federal (Possamai, 2016). Ao contrário do esperado com a criação do referido portal, contudo, os resultados deste estudo preliminar sugerem que usuários de dados abertos obtêm seus dados de modo descentralizado, por meio de buscas em diferentes portais, e utilizando mecanismos da transparência ativa e passiva.

O pesquisador entrevistado nesta pesquisa relatou, a título de exemplo, dificuldade em acessar uma base de dados para um estudo sobre migraçóes via transparência ativa, sendo que esta base só foi obtida via LAI.

Quando começaram a sair casos na mídia sobre migraçóes, todos foram em cima da Polícia Federal para buscar dados, muitas vezes pela Lei de Acesso à Informação. Seria muito mais fácil se esses dados já estivessem abertos e disponíveis. (...) Só conseguimos empreender uma ferramenta sobre migraçóes com dados solicitados pela LAI à Polícia Federal e depois de muito esforço e vários pedidos. Tem uma questão que é até cultural, de não entender a importância de se ter transparência (entrevistado 5).

\section{CONCLUSÃO E CONSIDERAÇÕES FINAIS}

Embora este estudo não seja suficiente para elencar os principais efeitos sociais, políticos e democráticos do uso de dados abertos governamentais no Brasil, foi possível lançar luz sobre alguns dos atores envolvidos nas apropriaçóes sociais desses dados. Em linhas gerais, os resultados apontam que há um ecossistema de usuários formado por partes interessadas de diferentes setores profissionais, sendo que os pesquisadores, gestores públicos, desenvolvedores e cientistas de dados afirmaram que dados abertos são especialmente relevantes para o desempenho de suas funçóes.

Apesar das dificuldades e barreiras elencadas, vimos, ainda, que os interessados nesses dados dialogam com diversos setores de atuação governamental e que os conjuntos de dados utilizados são adquiridos por meio de diferentes fontes e portais governamentais. Este mapeamento preliminar pode ser útil para a realização de consultas públicas e abertura de canais de comunicação com estes setores profissionais identificados como usuários de dados abertos no Brasil, para que políticas públicas e políticas de dados abertos sejam aprimoradas.

Ainda que se tenha a noção de que esse grupo de usuários é limitado em virtude das habilidades técnicas exigidas para a manipulação dos dados, argumenta-se aqui que, por meio da criação de iniciativas que utilizam como matéria-prima dados abertos - como reportagens jornalísticas, pesquisas, aplicativos e interfaces de visualização de dados -, cidadáos comuns são igualmente beneficiados com mais conhecimento, acesso à informaçáo e possibilidade de acompanhar e monitorar os negócios públicos, ainda que não sejam usuários primários desses dados. Esta pesquisa, de caráter exploratório, não foi capaz de elencar relaçôes de causalidade entre o uso de dados abertos e o fortalecimento de princípios democráticos. Todavia, argumenta-se que é possível esperar, pelo menos, que sejam fomentados a produção do conhecimento, o acompanhamento e monitoramento da açáo governamental e a produçáo de um jornalismo de melhor qualidade, que são, também, elementos importantes para as democracias contemporâneas.

Por fim, destaca-se que mais pesquisa é necessária para identificar e avaliar os tipos de usos e usuários de dados abertos no país. Há poucos estudos, por exemplo, que investigam iniciativas de dados abertos empreendidas por governos locais, pelo Legislativo e Judiciário, e que avaliam os usos e a qualidade dos dados publicados por esses entes públicos. 


\section{REFERÊNCIAS}

BAACK, S. Datafication and empowerment: how the open data movement re-articulates notions of democracy, participation, and journalism. Big Data and Society, v. 2, n. 2, p. 1-11, Dec. 2015.

BATES, J. "This is what modern deregulation looks like": co-optation and contestation in the shaping of the UK's open government data initiative. The Journal of Community Informatics, v. 8, n. 2, Apr. 2012.

CLARKE, A.; MARGETTS, H. Governments and citizens getting to know each other? Open, closed, and big data in public management reform. Policy and Internet, v. 6, n. 4, p. 393-417, 2014.

CONRADIE, P.; CHOENNI, S. On the barriers for local government releasing open data. Government Information Quarterly, v. 31, p. S10-S17, 2014. Supplement 1.

DE-LIMA-SANTOS, M. F. Tem \#DDJBR aqui? Mapeando a presença do jornalismo de dados no Brasil. In: CONGRESSO BRASILEIRO DE CIÊNCIAS DA COMUNICAÇÃO, 42., 2019, Belém, Pará. Anais... [s.l.]: Intercom, 2019. Disponível em: <http://portalintercom.org.br/anais/ nacional2019/resumos/R14-1396-1.pdf>.

EVANS, A. M.; CAMPOS, A. Open government initiatives: challenges of citizen participation. Journal of Policy Analysis and Management, v. 32, n. 1, p. 172-185, Jan. 2013.

GEIGER, C. P.; LUCKE, J. von. Open government and (linked) (open) (government) (data). JeDEM, v. 4, n. 2, p. 265-278, 2012.

GONZALEZ-ZAPATA, F.; HEEKS, R. The multiple meanings of open government data: understanding different stakeholders and their perspectives. Government Information Quarterly, v. 32, n. 4, p. 441-452, 2015.

HOWARD, A. Open data economy: eight business models for open data and insight from Deloitte UK. [s.l.]: [s.n.], 2013. Disponível em: <https://www.oreilly.com/ideas/open-data-business-modelsdeloitte-insight>. Acesso em: 22 jan. 2019.

JANSSEN, K. The influence of the PSI directive on open government data: an overview of recent developments. Government Information Quarterly, v. 28, n. 4, p. 446-456, 2011.

JANSSEN, M.; CHARALABIDIS, Y.; ZUIDERWIJK, A. Benefits, adoption barriers and myths of open data and open government. Information Systems Management, v. 29, n. 4, p. 258-268, 2012.

KITCHIN, R. The data revolution: big data, open data, data infrastructures and their consequences. [s.l.]: Sage Publications, 2014.

KUČERA, J.; CHLAPEK, D.; NEČASKÝ, M. Open government data catalogs: current approaches and quality perspective. In: KÖ, A. et al. (Ed.). Technology-enabled innovation for democracy, government and governance. Heidelberg: Springer, 2013. p. 152-166. (Lecture Notes in Computer Science, v. 8061).

LINDMAN, J. Similarities of open data and open source: impacts on business. Journal of Theoretical and Applied Electronic Commerce Research, v. 9, n. 3, p. 59-70, 2014.

LOURENÇO, R. P. An analysis of open government portals: a perspective of transparency for accountability. Government Information Quarterly, v. 32, n. 3, p. 323-332, 2015. 
NOVECK, B. S. Smart citizens, smarter state: the technologies of expertise and the future of governing. [s.l.]: Harvard University Press, 2015.

PINHO, M. D. C. Governo aberto e dados abertos governamentais: um estudo sobre as políticas e os usos de dados abertos governamentais no Brasil. 2019. 164 f. Dissertação (Mestrado) Universidade Federal da Bahia, Salvador, 2019. Disponível em: <http://poscom.tempsite.ws/wpcontent/uploads/2011/05/Dissertao_Maria_Dominguez_ultima_versao.pdf>.

POSSAMAI, A. J. Portal Brasileiro de Dados Abertos: novas práticas para o fortalecimento da democracia e da gestão pública na era digital. In: SILVA, S. P. da; BRAGATTO, R. C.; SAMPAIO, R. C. (Org.). Democracia digital, comunicaçáo política e redes: teoria e prática. 1. ed. Rio de Janeiro: Letra e Imagem, 2016, p. 365-392.

SAFAROV, I.; MEIJER, A.; GRIMMELIKHUIJSEN, S. Utilization of open government data: a systematic literature review of types, conditions, effects and users. Information Polity, v. 22, n. 1, p. 1-24, 2017.

SANDOVAL-ALMAZAN, R.; STYRIN, E. Towards an open government data comparative model. In: INTERNATIONAL CONFERENCE ON THEORY AND PRACTICE OF ELECTRONIC GOVERNANCE, 11., 2018, New York. Proceedings... New York: ACM Press, 2018. Disponível em: <http://dl.acm.org/citation.cfm?doid=3209415.3209434>.

ZELETI, F. A.; OJO, A.; CURRY, E. Exploring the economic value of open government data. Government Information Quarterly, v. 33, n. 3, p. 535-551, 2016.

ZUIDERWIJK, A. Open data infrastructures: the design of an infrastructure to enhance the coordination of open data use. Leidschendam: Uitgeverij BOXPress, 2015. Disponível em: <http:// repository.tudelft.nl/view/ir/uuid\%3A9b9e60bc-1edd-449a-84c6-7485d9bde012/>. 
